\title{
The asteroid-comet continuum from laboratory and space analyses of comet samples and micrometeorites
}

\author{
Cécile Engrand ${ }^{1}$, Jean Duprat ${ }^{1}$, Noémie Bardin $^{1}$, Emmanuel \\ Dartois $^{2}$, Hugues Leroux ${ }^{3}$, Eric Quirico ${ }^{4}$, Karim Benzerara ${ }^{5}$, Laurent \\ Remusat $^{5}$, Elena Dobrică ${ }^{6}$, Lucie Delauche ${ }^{1}$, John Bradley ${ }^{7}$, Hope \\ Ishii $^{7}$, Martin Hilchenbach ${ }^{8}$ and the COSIMA team \\ ${ }^{1}$ CSNSM, CNRS/IN2P3-Univ. Paris-Sud, Université Paris-Saclay, 91405 Orsay, France \\ email: cecile.engrand@csnsm.in2p3.fr \\ ${ }^{2}$ IAS, CNRS/INSU-Univ. Paris-Sud, Université Paris-Saclay, 91405 Orsay, France \\ ${ }^{3}$ UMET, CNRS/Univ. Lille1, 59655 Villeneuve d'Ascq, France \\ ${ }^{4}$ IPAG, UMR 5274 CNRS/INSU-UJF-Grenoble 1, 38041 Grenoble, France \\ ${ }^{5}$ IMPMC, MNHN, Case postale 115, 4 place Jussieu, 75252 Paris Cedex 05, France \\ ${ }^{6}$ Dpt Earth Planetary Sciences MSC03 2040, Univ. New Mexico, Albuquerque NM 87131, USA \\ ${ }^{7}$ Hawai'i Inst. Geophysics \& Planetology, University of Hawai'i, Honolulu, HI 96822, USA \\ ${ }^{8}$ Max-Planck-Institut für Sonnensystemforschung, 37077 Göttingen, Germany
}

\begin{abstract}
Comets are probably the best archives of the nascent solar system, 4.5 Gyr ago, and their compositions reveal crucial clues on the structure and dynamics of the early protoplanetary disk. Anhydrous minerals (olivine and pyroxene) have been identified in cometary dust for a few decades. Surprisingly, samples from comet Wild2 returned by the Stardust mission in 2006 also contain high temperature mineral assemblages like chondrules and refractory inclusions, which are typical components of primitive meteorites (carbonaceous chondrites - CCs). A few Stardust samples have also preserved some organic matter of comet Wild 2 that share some similarities with CCs. Interplanetary dust falling on Earth originate from comets and asteroids in proportions to be further constrained. These cosmic dust particles mostly show similarities with CCs, which in turn only represent a few percent of meteorites recovered on Earth. At least two (rare) families of cosmic dust particles have shown strong evidences for a cometary origin: the chondritic porous interplanetary dust particles (CP-IDPs) collected in the terrestrial stratosphere by NASA, and the ultracarbonaceous Antarctic Micrometeorites (UCAMMs) collected from polar snow and ice by French and Japanese teams. Analyses of dust particles from the Jupiter family comet $67 \mathrm{P} /$ Churyumov-Gerasimenko by the dust analyzers on Rosetta orbiter (COSIMA, GIADA, MIDAS) suggest a relationship to interplanetary dust/micrometeorites. A growing number of evidences highlights the existence of a continuum between asteroids and comets, already in the early history of the solar system.
\end{abstract}

Keywords. solar system: general, comets: general, minor planets, asteroids

\section{Introduction}

The structure and composition of the protoplanetary disk can be investigated by the study of the small bodies that have escaped planetary formation in the solar system: asteroids and comets. Samples from asteroid 25143 Itokawa returned by the Hayabusa mission and from comet $81 \mathrm{P} /$ Wild 2 returned by Stardust mission provide ground truth analyses for these small bodies (e.g. Nakamura et al. 2011; Brownlee 2014). Meteorites recovered on Earth can also give information on the formation and evolution of asteroids. 
Cosmic dust particles originate from both comets and asteroids, in proportions that are still debated. They can be efficiently collected in the stratosphere (as interplanetary dust particles - IDPs) by NASA (Brownlee 1985), and as micrometeorites (MMs) from snow and ice in polar caps (e.g. Maurette et al. 1991; Duprat et al. 2007; Taylor et al. 2000; Yada \& Kojima 2000). They can also be studied as microxenoliths trapped in meteorites (e.g. Bradley et al. 2014; Engrand et Maurette 1998; Briani et al. 2011). Analyses of comet $81 \mathrm{P} /$ Wild 2 (Stardust samples), meteorites and cosmic dust, as well as recent data obtained with the Rosetta mission on comet $67 \mathrm{P} /$ Churyumov-Gerasimenko suggest the presence of a continuum between asteroids and comets.

\section{The asteroid-comet continuum seen from space and laboratory analyses}

Carbonaceous chondrites (especially the CI type) have preserved the solar photosphere's composition for non volatile elements, and are taken as reference composition of the material initially present in the protosolar nebula (Lodders et al. 2010). They however have lost the most volatile elements, that are in turn preserved in comets. Carbonaceous chondrites are related to carbonaceous asteroids, but they represent only about $5 \%$ of the meteorites. A reexamination of the compositional classes in the main asteroid belt has shown that carbonaceous asteroids are actually present throughout the whole main belt, including the inner regions (DeMeo \& Carry 2014). Meteorites thus give a biased view of the types and abundances of interplanetary matter, as more than $80 \%$ of the meteorites are classified as ordinary chondrites that are related to S-type asteroids. This bias is probably due to preferential destruction of carbonaceous chondrites during atmospheric entry or by terrestrial weathering at the Earth's surface.

Comets contain crystalline Mg-rich silicates, olivines and pyroxenes, initially identified by infrared spectroscopy (e.g. Wooden et al. 2000; Hanner \& Zolensky 2010), and show a large variety of organic compounds (e.g. Bockelée-Morvan et al. 2004). Samples from comet $81 \mathrm{P} /$ Wild 2 (brought back by the Stardust mission in 2006) show significant similarities with carbonaceous chondrites (CCs): a chemical composition compatible (within a factor of 2) with that of CI chondrites, the presence of high temperature components: refractory inclusions (Ca-Al-rich inclusions or CAIs) and chondrule-like objects showing CC-like oxygen isotopic compositions, and remnant organics showing high level of complexity (Flynn et al. 2006; Zolensky et al. 2006; Nakamura et al. 2008; Nakashima et al. 2012; Ogliore et al. 2015; De Gregorio et al. 2011 and references therein). These results further outlined the need for radial transport from the inner regions of the protoplanetary disk where high temperature minerals are formed to the outer regions of the solar system where comets are stored (e.g. Bockelée-Morvan et al. 2002). They also showed for the first time a possible link between Jupiter family comet samples and carbonaceous chondrites - and therefore carbonaceous asteroids. Further Al-Mg dating of refractory components of $81 \mathrm{P} /$ Wild 2 samples (CAIs and chondrules) suggest a late formation of these components, constraining the formation age of Jupiter to be less than $3 \mathrm{Myr}$ after the formation of CAIs, as radial transport from the inner to the outer regions of the protoplanetary disk would be inhibited in the presence of Jupiter (Nakashima et al. 2015 and references therein).

It has also been proposed for a long time that some meteorites (or meteorite classes) could come from comets. These candidates mostly belong to the CI or CR groups (e.g. Anders 1975; McSween \& Weissman 1989; Campins \& Swindle 1998; Gounelle et al. 2004; Gounelle et al. 2008; Nakashima et al. 2012). These assumptions are based on orbital considerations, abundance of organic compounds (up to $5 \mathrm{wt} \%$ ), similarity in oxygen 
isotopes, elevated $\mathrm{D} / \mathrm{H}$ ratios or ${ }^{15} \mathrm{~N} /{ }^{14} \mathrm{~N}$ ratios, and abundance of glassy phases. Results from the Stardust mission cannot confirm (or not) the cometary origin of these meteorites.

Cosmic dust particles around $200 \mu \mathrm{m}$ currently represent the dominant input of extraterrestrial matter on Earth, and show similarities only with carbonaceous chondrites (less than $1 \%$ of the micrometeorites in that size range are related to ordinary chondrites). These cosmic dust particles probably originate from the outer regions of the asteroid belt and/or from the Jupiter family comets (JFCs) (Nesvorný et al. 2010). The characteristics of Stardust samples generally match those of micrometeorites and IDPs (e.g. Dobrică et al. 2009), in particular in terms of oxygen isotopes (Engrand et al. 1999; McKeegan et al. 1987; Aléon et al. 2009). Two relatively rare classes of cosmic dust particles collected on Earth have a very probable cometary origin: the chondritic-porous (CP) IDPs-MMs (Bradley et al. 2014; Noguchi et al. 2015), and the ultracarbonaceous Antarctic micrometeorites (UCAMMs) that show similarities with the CHON particles detected in comet 1P/Halley (Duprat et al. 2010; Nakamura et al. 2005). These particles are characterized by a high carbon content (with up to $80 \mathrm{vol} \%$ in the case of UCAMMs) showing anomalous (D-rich) hydrogen isotopic compositions, an anhydrous mineralogy showing a high abundance of pyroxene minerals with regard to olivines (while olivine is the most abundant anhydrous minerals in meteorites, with the exception of the CR clan), and the widespread occurence of glassy phases coined GEMS (Glass with Embedded Metals and Sulfides) that have a debated presolar origin (Keller \& Messenger 2013; Bradley 2013). These particles could be even more primitive than sample brought back by Stardust (e.g. Ishii et al. 2008). The organic matter of UCAMMs is enriched in nitrogen (with atomic $\mathrm{N} / \mathrm{C}$ ratios up to 0.2 ) and could have been formed by irradiation at the surface of a $\mathrm{CH}_{4}-\mathrm{N}_{2}$ rich icy body in the Oort cloud comet reservoir (Dartois et al. 2013).

One of the highlights of the Rosetta mission on comet $67 \mathrm{P} /$ Churyumov-Gerasimenko $(67 \mathrm{P} / \mathrm{C}-\mathrm{G})$ is the water $\mathrm{D} / \mathrm{H}$ ratio of $(5.3 \pm 0.7) \times 10^{-4}$, that is about 3 times the terrestrial value (Altwegg et al. 2015), a value for a JFC that is even higher than that measured for the Oort Cloud comet Hale-Bopp (Bockelée-Morvan et al. 2015). This result confirms the fact that $\mathrm{D} / \mathrm{H}$ (and ${ }^{15} \mathrm{~N} /{ }^{14} \mathrm{~N}$ ) ratios in comets are not characteristic of the comet reservoir. The D/H ratio measured in cometary HCN in Hale Bopp $\left((2.3 \pm 0.4) \times 10^{-3}\right)$, is compatible with that of organic matter in UCAMMs (Duprat et al. 2010). Analyses of dust particles with COSIMA on Rosetta show the association of rock forming elements with carbonaceous matter and highlights the enrichment of the dust particles in Na. They suggest similarities between the refractory (i.e. non-icy) component of $67 \mathrm{P} / \mathrm{C}-\mathrm{G}$ dust and cosmic dust collected on Earth (e.g. Schulz et al. 2015).

\section{Implications}

The analyses of samples from space missions (e.g. Stardust, Rosetta), of meteorites and cosmic dust highlight the existence of a continuum between primitive asteroids and comets. The presence of active asteroids main-belt comets also confirms the existence of this continuum. The presence of refractory minerals in comets invokes the need for radial transport from the inner to the outer regions of the protoplanetary disk, very early in the history of the solar system, and lasting at least a few Myr. Cosmic dust particles collected on Earth preferentially sample the carbonaceous asteroids and the cometary reservoir, thus being relevant samples to study this continuum. There is no clear evidence of large compositional differences between comets originating from the Kuiper Belt, and from the Oort cloud, supporting a common place for the formation of all comets. Comet samples are probably present in the terrestrial cosmic dust collections, in the form of 
chondritic porous IDPs collected in the stratosphere, and ultracarbonaceous Antarctic micrometeorites. The formation of N-rich organic matter of UCAMMs could result from irradiation of $\mathrm{CH}_{4}-\mathrm{N}_{2}$-rich surface of icy bodies, in the comet formation regions.

\section{References}

Aléon, J. et al. 2009, Geochim. Cosmochim. Acta 73, 4558-4575

Altwegg, K., et al. 2015, Science 347

Anders, E. 1975, Icarus 24, 363-371

Bockelée-Morvan, D. et al. 2002, A\&\&A 384, 1107-1118

Bockelée-Morvan, D. et al. 2004, In Comets II Univ. Arizona Press, pp. 391-423

Bockelée-Morvan, D., et al. 2015, Space Sci. Rev., 1-37

Bradley, J. P. 2014, In Treatise on Geochemistry (Second Edition), Elsevier, Oxford. pp. 287-308

Bradley, J. P. 2013, Geochim. Cosmochim. Acta 107, 336-340

Briani, G. et al. 2011, Meteoritics Planet. Sci. 46, 1863-1877

Brownlee, D.E. 1985 Ann. Rev. Earth Planet. Sci. 13, 147-173

Brownlee, D. E. 2014, Annual Review of Earth and Planetary Sciences 42, 179-205

Campins, H. \& Swindle, T. D. 1998, Meteoritics Planet. Sci. 33, 1201-1211

DeMeo, F. E. \& Carry, B. 2014, Nature 505, 629-634

Dartois, E. et al. 2013, Icarus 224, 243-252

De Gregorio, B. T. et al. 2011, Meteoritics Planet. Sci. 46, 1376-1396

Dobrică, E. et al. 2009, Meteoritics Planet. Sci. 44, 1643-1661

Duprat, J. et al. 2007, Adv. Space Res. 39, 605-611

Duprat, J. et al., 2010 Science 328, 742-745

Engrand, C. \& Maurette, M. 1998, Meteoritics Planet. Sci. 33, 565-580

Engrand, C., McKeegan, K. D., \& Leshin, L. A. 1999, Geochim. Cosmochim. Acta 63, 2623-2636

Flynn, G. J., et al. 2006, Science 314, 1731-1735

Gounelle, M., Spurny, P., \& Bland, P. A. 2004, Meteoritics Planet. Sci. 39, \#5174

Gounelle, M. et al. 2008, In The Solar System Beyond Neptune, Arizona Univ. Press. 525-541

Hanner, M. S. \& Zolensky, M. E. 2010, In Astromineralogy, Springer-Verlag. 203-226.

Ishii, H.A. et al., 2008 Science 319, 447-450.

Keller, L. P., Thomas, K. L., \& McKay, D. S. 1992, Geochim. Cosmochim. Acta 56, 1409-1412

Keller, L. P. \& Messenger, S. 2013, Geochim. Cosmochim. Acta 107, 341-344

Lodders, K. 2010, In Principles and Perspectives in Cosmochemistry(eds. A. Goswami and B. E. Reddy), Springer Berlin Heidelberg. pp. 379-417

Maurette, M. et al. 1991, Nature 351, 44-47

McKeegan, K. D. 1987, Science 237, 1468-1471

McSween, H. Y., Jr. \& Weissman, P. R. 1989, Geochim. Cosmochim. Acta 53, 3263-3271

Nakamura, T. et al. 2011, Science, 333, 113

Nakamura, T. et al. 2008, Science 321, 1664-1667

Nakamura, T. et al. 2005, Meteoritics Planet. Sci. 40 Suppl., \#5046

Nakashima, D. et al. 2012, Earth Planet. Sci. Lett. 357-358, 355-365

Nakashima, D. et al. 2015, Earth Planet. Sci. Lett. 410, 54-61

Nesvorný, D. et al. 2010, Astrophys. J. 713, 816-836

Noguchi, T. et al. 2015, Earth Planet. Sci. Lett. 410, 1-11

Ogliore, R. C. et al. 2015, Geochim. Cosmochim. Acta 166, 74-91

Schulz, R. et al. 2015, Nature 518, 216-218

Taylor, S., Lever, J. H., \& Harvey, R. P. 2000, Meteoritics Planet. Sci. 35, 651-666

Yada, T. \& Kojima, H. 2000, Antarctic Met. Res. 13, 9-18

Wooden, D. H., Butner, H. M., Harker, D. E., \& Woodward, C. E. 2000, Icarus 143, 126-137

Zolensky, M. E. et al. 2006, Science 314, 1735-1739 